\title{
A photonic basis for deriving nonlinear optical response
}

\author{
David L Andrews and David S Bradshaw \\ Nanostructures and Photomolecular Systems, School of Chemical Sciences, University of East \\ Anglia, Norwich NR4 7TJ, UK \\ E-mail: david.andrews@physics.org
}

Received 2 July 2008, in final form 27 November 2008

Published 5 January 2009

Online at stacks.iop.org/EJP/30/239

\begin{abstract}
Nonlinear optics is generally first presented as an extension of conventional optics. Typically the subject is introduced with reference to a classical oscillatory electric polarization, accommodating correction terms that become significant at high intensities. The material parameters that quantify the extent of the nonlinear response are cast as coefficients in a power seriesnonlinear optical susceptibilities signifying a propensity to generate optical harmonics, for example. Taking the subject to a deeper level requires a more detailed knowledge of the structure and properties of each nonlinear susceptibility tensor, the latter differing in form according to the process under investigation. Typically, the derivations involve intricate development based on time-dependent perturbation theory, assisted by recourse to a set of Feynman diagrams. This paper presents a more direct route to the required results, based on photonic rather than semiclassical principles, and offers a significantly clearer perspective on the photophysics underlying nonlinear optical response. The method, here illustrated by specific application to harmonic generation and down-conversion processes, is simple, intuitive and readily amenable for processes of arbitrary photonic order.
\end{abstract}

\section{Introduction}

The field of nonlinear optics is one of the most striking developments to have emerged from the development of the laser almost 50 years ago. Optical frequency doubling (second-harmonic generation), for example, which produces tightly collimated emission in non-centrosymmetric crystals, rapidly proved its worth as a means of converting laser output to a shorter wavelength. The basic principles of this and other such nonlinear phenomena were rapidly established and consolidated, notably in Bloembergen's treatise (initially published in 1965) [1]. Since then, a rapid growth of diverse applications, including spectroscopic sensing, optical communications 
and signal processing have established and underscored the technical significance of nonlinear optical response. There are several modern introductions to the subject, which include useful texts by Butcher and Cotter [2], Jha [3], Sauter [4], He and Liu [5], Shen [6], Sutherland [7], Banerjee [8], Novotny and Hecht [9] and Boyd [10], though most are set at a level that quickly transcends the material appropriate for newly introducing students to the field.

A common device to explain the onset of optical nonlinearity is to make reference to a classical electric polarization, expanded as a power series in the oscillatory input field. The material parameters that enter the correction terms quantify the extent of the nonlinear response available from a given medium; nonlinear optical susceptibilities signify a propensity to generate optical harmonics, for example. This is the approach presented in many standard student textbooks [11-14]. To develop the subject at a deeper level, it is necessary to furnish other methods to elicit the detailed structure and properties of each nonlinear susceptibility tensor, the latter differing in its form according to the process under investigation. Typically, such derivations involve an intricate development based on time-dependent perturbation theory, usually assisted by Feynman or analogous diagrams. Such methods can nonetheless prove somewhat arduous even for a relatively simple process such as frequency doubling, and they acquire an ungainly length when applied to common three- or four-wave processes.

This paper presents a more direct and transparent route to the required results, based on sound photonic rather than semiclassical principles. Suitable for delivery to advanced physics majors, the derivations based on quantum electrodynamics (QED) are developed using an intuitive quantum state-sequence method, offering what we believe is a significantly clearer perspective on the photophysics underlying nonlinear optical response. The method, specifically illustrated by application to second and third-harmonic generation and parametric down-conversion, offers clear insights into the structures of the nonlinear optical property tensors involved. Extensions to processes of arbitrary photonic order are straightforward. We advocate the following described methods as a means of consolidating and deepening the understanding of optical nonlinearity at a senior undergraduate or graduate level-the only prerequisite being a general familiarity with quantum mechanics and nonlinear optics. The technique represents an obvious scope for practice exercises, and also provides an obvious basis for research projects leading to the forefront of current nonlinear optics.

\section{Semiclassical theory versus QED}

The proper description of any optical medium in atomic or molecular terms entails modelling its electronic behaviour under the influence of electromagnetic radiation, generally requiring a full quantum mechanical treatment of the material. There are, however, two distinct approaches to the detailed theoretical representation of optical processes at this level: for the radiation there exists the possibility of a classical description; the other possibility is a quantum mechanical development. The former choice, representing what is usually known as a semiclassical formulation of the theory, has the strength of relating clearly to classical electrodynamics and it is successful within a limited province. The alternative procedure where both matter and radiation are treated quantum mechanically, and which gives legitimacy to the concept of the photon, is known as quantum electrodynamics [15-18].

A semiclassical electrodynamical introduction to nonlinear optics generally subscribes to a fairly standard treatment. It is commonly shown how the linear response to an applied electric field, considered responsible for the induction of a dipole moment, represents only an approximation to the true response of many materials. When such a field is oscillatory, as in 
the optical case, it is reasoned that the correction terms associated with nonlinear response are responsible for the generation of optical harmonics, i.e. [1],

$$
P_{i}=\varepsilon_{0}\left[\sum_{j} \chi_{i j}^{(1)} E_{j}+\sum_{j, k} \chi_{i j k}^{(2)} E_{j} E_{k}+\sum_{j, k, l} \chi_{i j k l}^{(3)} E_{j} E_{k} E_{l}+\cdots,\right],
$$

where vector $\mathbf{P}$ is the electric polarization, $\mathbf{E}$ is the applied electric field and $\chi^{(q)}$ is the $q$ thorder electric susceptibility with the status of $(q+1)$ rank, as denoted by the number of indices. Furthermore, $i, j, k$ and $l$ signify Cartesian components $x, y$ and $z$; by adopting the convention of implied summation over repeated indices, the summation representations can be omitted in the following. From the semiclassical perspective, the electromagnetic radiation is considered a wave varying sinusoidally with time $t$, namely,

$$
E(t)=E_{0} \cos \omega t .
$$

With the use of equation (2), the expression of (1) may be recast as

$$
\begin{aligned}
P_{i}(t)=\chi_{i j}^{(1)} E_{0 j} & \cos \omega t+\frac{1}{2} \chi_{i j k}^{(2)} E_{0 j} E_{0 k}(1+\cos 2 \omega t) \\
& +\frac{1}{4} \chi_{i j k l}^{(3)} E_{0 j} E_{0 k} E_{0 l}(3 \cos \omega t+\cos 3 \omega t)+\cdots .
\end{aligned}
$$

The first correction term to the linear response, i.e., the second term of equation (3), is thus considered the source of an optical second harmonic (usually shying away from any discussion of its additional time-independent term); the next correction is deemed the origin of a third harmonic, and so on. From any such representation, it is simple enough to verify that the even harmonics are forbidden in centrosymmetric media (since the polarization must change sign on space inversion, whereas the responsible term in (3) does not). However, to derive the detailed structure of the linear and nonlinear susceptibilities, one usually has recourse to essentially prescriptive methods in a form first proposed by Ward [19], based on time orderings. Arguably, such methods afford little assistance to the development of fundamental understanding.

There are many reasons why the above classical description is unsatisfactory-as are delineated elsewhere [20, 21]. To illustrate, equation (3) logically implies that a medium with a non-vanishing $\chi^{(2)}$ will always generate some amount of second harmonic, even at intensity levels that would correspond to only one photon of input-whereas of course two input photons are required for every second-harmonic photon that is produced. The mistake owes its origin to the misrepresentation of the optical field by a classical wave-a description that makes no provision for accommodating quantized energy levels. It is, in fact, simple and instructive to show that the statistical probability of two photons arriving together leads to the observed quadratic dependence on intensity [22].

Here we set forth a more satisfactory, lucid and rigorous, photonic foundation for nonlinear optical phenomena, based on quantum electrodynamics (QED). The arguments for using a QED representation are indeed significantly more persuasive in the case of optically parametric processes, such as harmonic generation, than in the description of providing for electronic excitation in a medium. Parametric processes are specifically those in which real transitions take place between photon number states, but only virtual (non-energy conserving) transitions occur in the material medium. Energy is, of course, conserved in the overall process. We begin from the electric dipole interaction Hamiltonian, $H_{\text {int }}$, for an atom or molecule at position $\mathbf{R}$. This operator is the scalar product of the transverse electric displacement field, $\mathbf{d}^{\perp}(\mathbf{R})$ (the radiation operator) and the electric dipole moment operator, $\boldsymbol{\mu}$ (the matter operator) - the full expression is written as

$$
H_{\text {int }}=-\varepsilon_{0}^{-1} \boldsymbol{\mu} \cdot \mathbf{d}^{\perp}(\mathbf{R}) .
$$


The quantum amplitude $M_{f i}$ which signifies the coupling between initial and final statescontaining information on both the matter and radiation-is determined by time-dependent perturbation theory [23]. The result is generally cast as the following infinite series:

$$
\begin{aligned}
M_{f i}= & \left\langle F\left|H_{\mathrm{int}}+\sum_{p=1}^{\infty}\left\{H_{\mathrm{int}}\left(T_{0} H_{\mathrm{int}}\right)^{p}\right\}\right| I\right\rangle \\
= & \left\langle F\left|H_{\mathrm{int}}+H_{\mathrm{int}} T_{0} H_{\mathrm{int}}+H_{\mathrm{int}} T_{0} H_{\mathrm{int}} T_{0} H_{\mathrm{int}}+H_{\mathrm{int}} T_{0} H_{\mathrm{int}} T_{0} H_{\mathrm{int}} T_{0} H_{\mathrm{int}}+\ldots\right| I\right\rangle \\
= & \left\langle F\left|H_{\mathrm{int}}\right| I\right\rangle+\sum_{R} \frac{\left\langle F\left|H_{\mathrm{int}}\right| R\right\rangle\left\langle R\left|H_{\mathrm{int}}\right| I\right\rangle}{\left(E_{I}-E_{R}\right)}+\sum_{R, S} \frac{\left\langle F\left|H_{\mathrm{int}}\right| S\right\rangle\left\langle S\left|H_{\mathrm{int}}\right| R\right\rangle\left\langle R\left|H_{\mathrm{int}}\right| I\right\rangle}{\left(E_{I}-E_{R}\right)\left(E_{I}-E_{S}\right)} \\
& +\sum_{R, S, T} \frac{\left\langle F\left|H_{\mathrm{int}}\right| T\right\rangle\left\langle T\left|H_{\mathrm{int}}\right| S\right\rangle\left\langle S\left|H_{\mathrm{int}}\right| R\right\rangle\left\langle R\left|H_{\mathrm{int}}\right| I\right\rangle}{\left(E_{I}-E_{R}\right)\left(E_{I}-E_{S}\right)\left(E_{I}-E_{T}\right)}+\cdots,
\end{aligned}
$$

where $|I\rangle$ and $|F\rangle$ represent the initial and final system states, respectively, and $T_{0} \approx$ $\left(E_{I}-H_{0}\right)^{-1}$. In the latter, $H_{0}$ is the unperturbed Hamiltonian that operates on the virtual intermediate states, denoted by $|R\rangle,|S\rangle,|T\rangle \ldots$ and $E_{I}$ is the energy of the initial state; each denominator term in equation (5) is a difference between state energies. Moreover, successive terms relate to processes of progressively higher photonic order; for example, a description of the three-photon event of second-harmonic generation (SHG) and four-photon event of third-harmonic generation (THG) are determined from the third and fourth terms on the righthand side of equation (5), respectively. Under conditions where optical field strengths are not sufficiently high to disrupt the material, equation (5) converges and higher order terms represent signals of diminishing magnitude. To determine an experimentally quantifiable variable from equations (3) and (5) usually involves finding their modulus squared. In the semiclassical description a problem arises since the resulting cross-terms have no practical significance. In contrast, processes cannot involve both three and four photons (for example) and, thus, no cross-terms will occur in the QED case.

\section{Quantum interactions}

In a recent report [24] the formal basis was developed for a graph theoretic approach for computations, based on the development of terms in the expansion (5), and leading to full expressions for the nonlinear optical susceptibilities. The method, which involves the identification of quantum channels (signifying quantum mechanically distinct routes connecting initial and final states), entails the construction of a graph that affords any photonic process a unique and complete representation. Each such graph can be interpreted as a state-sequence network connecting the initial and final system states- the physical system comprising both molecular and radiation components. Any pathway through such a graph represents a quantum channel, each generating a directly additive contribution to the nonlinear response tensor. Since its inception and first application [25] this method has widely been tested, its calculational efficiency proved and results are already reported for a diverse range of optical applications [26-29].

\subsection{State-sequence diagrams}

The method we advocate is based on a simple and intuitive representation of photon state transitions. Since, in any order of multipolar interaction, electromagnetic field operations can only effect the creation or annihilation of a single photon, the states involved in any process coupling $N$ radiation modes can be presented as a sequence of translations between integer 


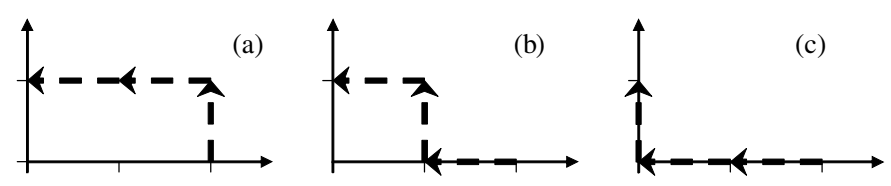

Figure 1. Three routes from the initial to final state, employing the photonic basis set $\left|n_{\omega}, n_{\omega^{\prime}}\right\rangle$, vertex sequences as follows: (a) $|2,0\rangle \rightarrow|2,1\rangle \rightarrow|1,1\rangle \rightarrow|0,1\rangle$; (b) $|2,0\rangle \rightarrow|1,0\rangle \rightarrow|1,1\rangle \rightarrow$ $|0,1\rangle ;$ (c) $|2,0\rangle \rightarrow|1,0\rangle \rightarrow|0,0\rangle \rightarrow|0,1\rangle$.

points in $\mathrm{N}$-space. This is simplest to illustrate by reference to second-harmonic generation (SHG), where only $N=2$ radiation modes need to be considered; an input of frequency $\omega$, say, and an output $\omega^{\prime}=2 \omega$. The input laser mode initially has $n_{\omega}=2$ photons of the fundamental frequency; the process of SHG removes these and delivers one photon into the harmonic frequency mode resulting in $n_{\omega^{\prime}}=1$. The radiation part of the initial and final states, expressible as members of a photonic set $\left|n_{\omega}, n_{\omega^{\prime}}\right\rangle$, can thus be represented as $|2,0\rangle$ and $|0,1\rangle$, and the three possible routes between them-i.e., the three quantum channels-are shown as tracks in 2D space in figure 1. Each corresponds to a different radiation state sequence. One immediate advantage of the representation in figure 1, exploited in the development below, is the ready identification of states $|1,0\rangle$ and $|1,1\rangle$ as intermediates that feature in more than one channel; the former viewed within figures 1(b) and (c), the latter in figures 1(a) and (b). To this end, all three tracks in figure 1 can be superimposed, so that the three state sequences are illustrated in a single diagram.

Many nonlinear optical processes (including all orders of harmonic generation) entail more than one interaction having the same effect on radiation mode occupancy-such as the two interchangeable photon annihilations in the second-harmonic case. As a result of such degeneracy, the number of interactions that are distinct in the photonic basis is generally less than the total number. Each distinct type of coupling can be represented by a unit vector $\hat{i}_{j}$ in an $N$-dimensional space, where $N$ is the number of distinct types of interaction. Each vector can also be assigned a coefficient $c_{j}$ denoting the number of occurrences of their respective interactions. Any nonlinear optical process can thus be coded by a set $I$ of these vectors as follows:

$$
I=\left\{c_{1} \hat{\boldsymbol{i}}_{1}, c_{2} \hat{\boldsymbol{i}}_{2}, \ldots, c_{N} \hat{\boldsymbol{i}}_{N}\right\}
$$

where the vectors have a designated ordering such that $c_{1} \leqslant c_{2} \leqslant \cdots \leqslant c_{N}$. Again, with reference to the case of second-harmonic generation (SHG), the annihilation of two photons with frequency $\omega$ and emission of another with frequency $\omega^{\prime}=2 \omega$ is represented by the vector set:

$$
I^{\mathrm{SHG}}=\left\{1 \hat{\boldsymbol{i}}_{1}, 2 \hat{\boldsymbol{i}}_{2}\right\}
$$

This representation corresponds to a third-order process embedded in 2-space, as denotes a photon creation and each of two identical annihilation events. Returning to the three state sequences of figure 1 superimposed upon one another, each node can be assigned a coordinate $\left(C_{1}, C_{2}\right)$-where $C_{1}$ and $C_{2}$ are the numbers of occurrences of $\hat{i}_{1}$ and $\hat{i}_{2}$, respectively, that have occurred between the initial state and the state represented by that node. Therefore, the initial and final states of SHG are represented by $(0,0)$ and $(1,2)$, and the intermediate states may be similarly defined. Mapping this coordinate system into base $B=c_{N}+1$, the following general expression is determined:

$$
\left.\left(C_{1}, C_{2}, \ldots, C_{N}\right) \rightarrow C_{1} C_{2} \ldots C_{N}\right|_{B} \Rightarrow h
$$


Table 1. Properties of the nodes within figures 1-3.

\begin{tabular}{|c|c|c|c|}
\hline$k$ & Coordinate & $\begin{array}{l}\text { String number } \\
\text { (base } B=3 \text { ) }\end{array}$ & $h$ \\
\hline 0 & $(0,0)$ & 00 & 0 \\
\hline 1 & $(0,1)$ & 01 & 1 \\
\hline 1 & $(1,0)$ & 10 & 3 \\
\hline 2 & $(0,2)$ & 02 & 2 \\
\hline 2 & $(1,1)$ & 11 & 4 \\
\hline 3 & $(1,2)$ & 12 & 5 \\
\hline
\end{tabular}

Table 2. All states and their associated energies for the state sequences exhibited in figure 3.

\begin{tabular}{lll}
\hline System state $\left|r_{h}\right\rangle$ & $\left|A_{k} ; n_{\omega}, n_{\omega^{\prime}}\right\rangle$ & Energy $E_{r_{h}}$ \\
\hline$|I\rangle=\left|r_{0}\right\rangle$ & $\left|A_{0} ; 2,0\right\rangle$ & $E_{0}+2 \hbar \omega$ \\
$\left|r_{1}\right\rangle$ & $\left|A_{r} ; 1,0\right\rangle$ & $E_{r}+\hbar \omega$ \\
$\left|r_{3}\right\rangle$ & $\left|A_{r} ; 2,1\right\rangle$ & $E_{r}+4 \hbar \omega$ \\
$\left|r_{2}\right\rangle$ & $\left|A_{s} ; 0,0\right\rangle$ & $E_{s}$ \\
$\left|r_{4}\right\rangle$ & $\left|A_{s} ; 1,1\right\rangle$ & $E_{s}+3 \hbar \omega$ \\
$|F\rangle=\left|r_{5}\right\rangle$ & $\left|A_{0} ; 0,1\right\rangle$ & $E_{0}+2 \hbar \omega$ \\
\hline
\end{tabular}

where the right-hand side is not a product, but a string of characters to be read as a number in the base $B$ conveniently re-expressed as the corresponding number $h$ in a decimal basis-see below. Also of importance is the step number, $k$, which is defined by

$$
k=\sum_{j=1}^{N} C_{j}
$$

Using equations (8) and (9) the variables $k$ and $h$ are determined for each state (i.e., a node within the superimposed diagram) of SHG and are reported in table 1. Employing these two variables a three-interaction plane network is constructed for SHG (figure 2). The intermediate molecular states are established from this $k$ value and, thus, the earlier photonic set may be expanded to the complete set $\left|A_{k} ; n_{\omega}, n_{\omega^{\prime}}\right\rangle$. Hence, the initial $(h=0)$ and final states $(h=5)$ are now represented as $\left|A_{0} ; 2 ; 0\right\rangle$ and $\left|A_{3} ; 0 ; 1\right\rangle$, respectively. The latter is of course equivalent to $\left|A_{0} ; 0 ; 1\right\rangle$, by conservation of energy. The intermediate states (and their corresponding energies) are given in table 2; for clarity in the following, the subscript $k=$ $1,2 \ldots$ is now recast as $r, s \ldots$ to denote the first, second ... molecular intermediate states.

Following from the network plane of figure 2 and the complete set of each system state (a node on the figure), a state-sequence diagram is constructed (figure 3). Tracing through the diagram of figure 3 from the initial (on the left) to the final state (right) the earlier three routes are identified, each signifying a different sequence of fundamental $H_{\text {int }}$ operations. These state-sequence diagrams are the graphical implementation of a network representing system states distinguished by radiation mode occupation, and their connectivity. Each state is again signified by a node in the diagram and couplings between these states are symbolized by connecting lines or edges. When coupling is of electric dipole form, it follows from equations (4) and (5) that the edges must connect states that differ by exactly one photon (indeed, this is a rule that applies to electric or magnetic multipole coupling of any order). However, no restriction is imposed on the molecular states that are so coupled. 


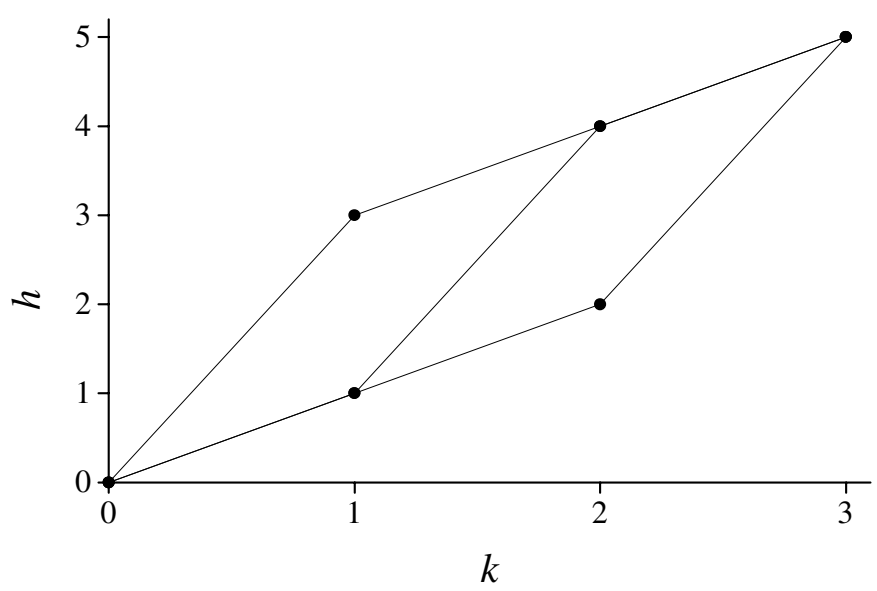

Figure 2. The three-interaction plane network for second-harmonic generation.

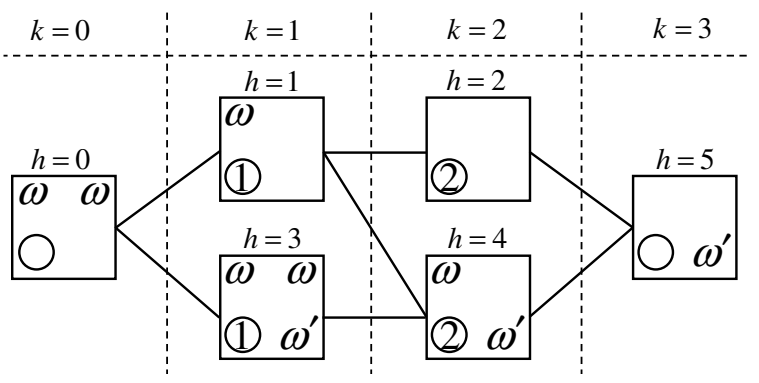

Figure 3. State-sequence diagram for second-harmonic generation. Two photons from mode $\omega$ are annihilated and one of mode $\omega^{\prime}$ is created. An unfilled circle represents a ground state, numbered circles an intermediate state of the optical centre.

\subsection{Adjacency matrices}

To determine the detailed form of optical susceptibility, using these state-sequence diagrams, an adjacency matrix method is applied. Such matrices are a commonly employed representation of the connectivity between nodes in a network [30]. For our purposes, a connectivity matrix, $M_{C}$-a molecular state projection of $H_{\text {int }}$ may generally be written as

$$
M_{C}=\sum_{\substack{h_{p}, h_{q}=0, h_{p} \neq h_{q}}}^{h_{\max }} \sum_{j=1}^{N}\left|h_{p}\right\rangle \hat{\boldsymbol{i}}_{j}\left\langle h_{q}\right|,
$$

where $h_{p}$ and $h_{q}$ are the indices of two nodes that are directly connected, self-interactions being excluded (i.e., $h_{p}=h_{q}$ ). For SHG, $j=1$ or 2 and the non-zero summands are those for which $\Delta h=h_{q}-h_{p}=3$ (corresponding to an emission, $\hat{\boldsymbol{i}}_{1}$ ) or 1 (absorption, $\hat{\boldsymbol{i}}_{2}$ ), respectively. Explicitly, for this case, equation (10) becomes

$M_{C}=|0\rangle \hat{\boldsymbol{i}}_{2}\langle 1|+| 0\rangle \hat{\boldsymbol{i}}_{1}\langle 3|+| 1\rangle \hat{\boldsymbol{i}}_{2}\langle 2|+| 1\rangle \hat{\boldsymbol{i}}_{1}\langle 4|+| 2\rangle \hat{\boldsymbol{i}}_{1}\langle 5|+| 3\rangle \hat{\boldsymbol{i}}_{2}\langle 4|+| 4\rangle \hat{\boldsymbol{i}}_{2}\langle 5|$.

Expressed in this projection form, the matrix represents all (forward) single steps between nodes in the state-sequence network of figure 3. It is possible to identify all the routes or quantum channels from the initial to final state through $M_{C}^{3}$ (since this is a three-interaction 
process). The following concise result is found:

$$
M_{C}^{3}=\hat{\boldsymbol{i}}_{2} \hat{\boldsymbol{i}}_{2} \hat{\boldsymbol{i}}_{1}+\hat{\boldsymbol{i}}_{2} \hat{\boldsymbol{i}}_{1} \hat{\boldsymbol{i}}_{2}+\hat{\boldsymbol{i}}_{1} \hat{\boldsymbol{i}}_{2} \hat{\boldsymbol{i}}_{2}=\mu_{k}^{r 0} \mu_{j}^{s r} \mu_{i}^{0 s}+\mu_{k}^{r 0} \mu_{i}^{s r} \mu_{j}^{0 s}+\mu_{i}^{r 0} \mu_{k}^{s r} \mu_{j}^{0 s},
$$

where $\hat{\boldsymbol{i}}_{1}$ designates a transition dipole moment component $\mu_{i}$ (the subscript $i$ is chosen to denote an emission) and $\hat{\boldsymbol{i}}_{2}$ to either $\mu_{j}$ or $\mu_{k}$. This expression is a representation of the order in which the interactions occur (signified by the superscripts $r 0, s r$ then $0 s$ ), the three terms representing the routes through figure 3. Furthermore, the change in the molecular state due to an interaction is denoted by the superscript deriving from a notation that follows from equations (4) and (5)—for example, $\boldsymbol{\mu}^{0 s}=\langle 0|\boldsymbol{\mu}| s\rangle$.

Equation (12) sums the numerators of the sought result. To ascertain the energy denominator for each of these terms (see equation (5)), a further matrix, which includes the system energetics, is required. This is generally given by

$$
M_{E}=\sum_{h=1}^{h_{\max }-1}|h\rangle S_{h}\langle h|,
$$

where $S_{h}=\left(E_{0}-E_{r_{h}}\right)^{-1}$, i.e., an eigenvalue of $T_{0}$, which is determined from table 2. By the multiplication of the matrices in the sequence $M_{C} M_{E} M_{C} M_{E} M_{C}$, an order congruent to the third term of the second line of (5), equation (12) becomes

$$
\begin{aligned}
M_{C} M_{E} M_{C} M_{E} M_{C}= & \hat{\boldsymbol{i}}_{2} S_{1} \hat{\boldsymbol{i}}_{2} S_{2} \hat{\boldsymbol{i}}_{1}+\hat{\boldsymbol{i}}_{2} S_{1} \hat{\boldsymbol{i}}_{1} S_{4} \hat{\boldsymbol{i}}_{2}+\hat{\boldsymbol{i}}_{1} S_{3} \hat{\boldsymbol{i}}_{2} S_{4} \hat{\boldsymbol{i}}_{2} \\
= & \sum_{r, s}\left(\frac{\mu_{i}^{0 s} \mu_{j}^{s r} \mu_{k}^{r 0}}{\left(E_{s 0}-2 \hbar \omega\right)\left(E_{r 0}-\hbar \omega\right)}+\frac{\mu_{j}^{0 s} \mu_{i}^{s r} \mu_{k}^{r 0}}{\left(E_{s 0}+\hbar \omega\right)\left(E_{r 0}-\hbar \omega\right)}\right. \\
& \left.+\frac{\mu_{j}^{0 s} \mu_{k}^{s r} \mu_{i}^{r 0}}{\left(E_{s 0}+2 \hbar \omega\right)\left(E_{r 0}+\hbar \omega\right)}\right) \\
= & \chi_{i j k}^{(2)}\left(\omega^{\prime} ; \omega, \omega\right) .
\end{aligned}
$$

This is the formal expression for the second-order optical susceptibility, $\chi_{i j k}^{(2)}\left(\omega^{\prime} ; \omega, \omega\right)$, of SHG. To cast this expression into standard SI units simply requires multiplication by a factor of $\varepsilon_{0} / \rho$, where $\rho$ is the number density of molecules.

\section{Further examples}

\subsection{Third-harmonic generation}

In contrast to SHG, third-harmonic generation (THG) entails the annihilation of three photons of frequency $\omega$ and emission of a photon with frequency $\omega^{\prime \prime}=3 \omega$, in a four-interaction process represented by the vector set $I^{\mathrm{THG}}=\left\{1 \hat{\boldsymbol{i}}_{1}, 3 \hat{\boldsymbol{i}}_{2}\right\}$. Following an analogous procedure to that described in the last section, the derivation begins with the construction of a state-sequence diagram for THG; to this end, the coordinate of each node, which corresponds to a system state, and the related $k$ and $h$ values are determined (table 3). The initial, intermediate and final states (and their corresponding energies) are given in table 4 , and the resulting state-sequence diagram is presented in figure 4 .

To derive the third-order susceptibility, $\chi_{i j k l}^{(3)}\left(\omega^{\prime \prime} ; \omega, \omega, \omega\right)$, we return to the general expression of (10) which, for this case, will again involve $j$ equating to either 1 or 2 -the non-zero summands are those for which $\Delta h=4$ (an emission, $\hat{i}_{1}$ ) or 1 (absorption, $\hat{\boldsymbol{i}}_{2}$ ), respectively-so that the equation is explicitly rewritten as

$$
\begin{aligned}
M_{C}=|0\rangle \hat{\boldsymbol{i}}_{2}\langle 1|+| 0\rangle \hat{\boldsymbol{i}}_{1}\langle 4|+| 1\rangle \hat{\boldsymbol{i}}_{2}\langle 2|+| 1\rangle \hat{\boldsymbol{i}}_{1}\langle 5|+| 2\rangle \hat{\boldsymbol{i}}_{2}\langle 3|+| 2\rangle \hat{\boldsymbol{i}}_{1}\langle 6|+| 4\rangle \hat{\boldsymbol{i}}_{2}\langle 5| \\
+|3\rangle \hat{\boldsymbol{i}}_{1}\langle 7|+| 5\rangle \hat{\boldsymbol{i}}_{2}\langle 6|+| 6\rangle \hat{\boldsymbol{i}}_{2}\langle 7| .
\end{aligned}
$$




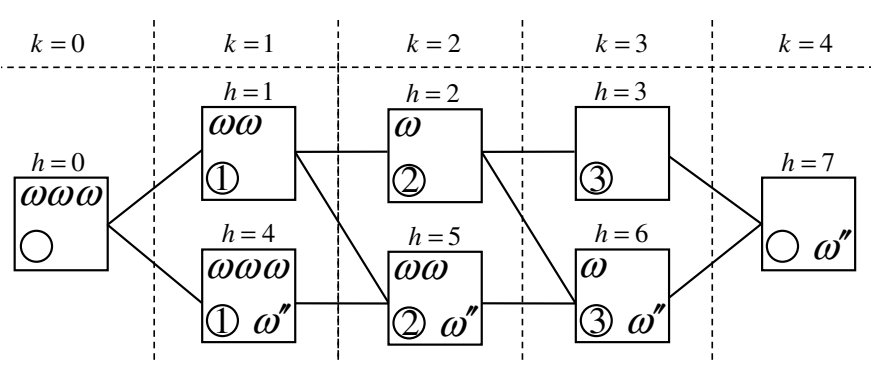

Figure 4. State-sequence diagram for third-harmonic generation. As figure 3, but here three photons of mode $\omega$ are annihilated and one is created with $\omega^{\prime \prime}$.

Table 3. Parameters employed in the construction of figure 4.

\begin{tabular}{|c|c|c|c|}
\hline$k$ & Coordinate & $\begin{array}{l}\text { String number } \\
\text { (base } B=4 \text { ) }\end{array}$ & $h$ \\
\hline 0 & $(0,0)$ & 00 & 0 \\
\hline 1 & $(0,1)$ & 01 & 1 \\
\hline 1 & $(1,0)$ & 10 & 4 \\
\hline 2 & $(0,2)$ & 02 & 2 \\
\hline 2 & $(1,1)$ & 11 & 5 \\
\hline 3 & $(0,3)$ & 03 & 3 \\
\hline 3 & $(1,2)$ & 12 & 6 \\
\hline 4 & $(1,3)$ & 13 & 7 \\
\hline
\end{tabular}

Table 4. All states and their associated energies for the state sequences exhibited in figure 4.

\begin{tabular}{lll}
\hline System state $\left|r_{h}\right\rangle$ & $\left|A_{k} ; n_{\omega}, n_{\omega^{\prime \prime}}\right\rangle$ & Energy $E_{r_{h}}$ \\
\hline$|I\rangle=\left|r_{0}\right\rangle$ & $\left|A_{0} ; 3,0\right\rangle$ & $E_{0}+3 \hbar \omega$ \\
$\left|r_{1}\right\rangle$ & $\left|A_{1} ; 2,0\right\rangle$ & $E_{r}+2 \hbar \omega$ \\
$\left|r_{4}\right\rangle$ & $\left|A_{1} ; 3,1\right\rangle$ & $E_{r}+6 \hbar \omega$ \\
$\left|r_{2}\right\rangle$ & $\left|A_{2} ; 1,0\right\rangle$ & $E_{s}+\hbar \omega$ \\
$\left|r_{5}\right\rangle$ & $\left|A_{2} ; 2,1\right\rangle$ & $E_{s}+5 \hbar \omega$ \\
$\left|r_{3}\right\rangle$ & $\left|A_{3} ; 0,0\right\rangle$ & $E_{t}$ \\
$\left|r_{6}\right\rangle$ & $\left|A_{3} ; 1,1\right\rangle$ & $E_{t}+4 \hbar \omega$ \\
$|F\rangle=\left|r_{7}\right\rangle$ & $\left|A_{0} ; 0,1\right\rangle$ & $E_{0}+3 \hbar \omega$ \\
\hline
\end{tabular}

Expressed in this projection form, the matrix represents all (forward) single steps between nodes in the state-sequence network of figure 4 . To determine all the routes from the initial to final state equation (15) must be quadrupled, since this and only this relates to a four-step process. Therefore, the following is found:

$$
\begin{aligned}
M_{C}^{4}=\hat{\boldsymbol{i}}_{2} \hat{\boldsymbol{i}}_{2} \hat{\boldsymbol{i}}_{2} \hat{\boldsymbol{i}}_{1}+\hat{\boldsymbol{i}}_{2} \hat{\boldsymbol{i}}_{2} \hat{\boldsymbol{i}}_{1} \hat{\boldsymbol{i}}_{2}+\hat{\boldsymbol{i}}_{2} \hat{\boldsymbol{i}}_{1} \hat{\boldsymbol{i}}_{2} \hat{\boldsymbol{i}}_{2}+\hat{\boldsymbol{i}}_{1} \hat{\boldsymbol{i}}_{2} \hat{\boldsymbol{i}}_{2} \hat{\boldsymbol{i}}_{2} \\
\quad=\mu_{l}^{r 0} \mu_{k}^{s r} \mu_{j}^{t s} \mu_{i}^{0 t}+\mu_{l}^{r 0} \mu_{k}^{s r} \mu_{i}^{t s} \mu_{j}^{0 t}+\mu_{l}^{r 0} \mu_{i}^{s r} \mu_{k}^{t s} \mu_{j}^{0 t}+\mu_{i}^{r 0} \mu_{l}^{s r} \mu_{k}^{t s} \mu_{j}^{0 t},
\end{aligned}
$$

where $\hat{\boldsymbol{i}}_{1}$ corresponds to the emission dipole moment $\mu_{i}$ and $\hat{\boldsymbol{i}}_{2}$ to either $\mu_{j}, \mu_{k}$ or $\mu_{l}$. The four terms of equation (16) correspond to the four routes through figure 4 . To ascertain the denominator for each of these terms a further matrix, which includes the system states energetics, is required. Again this is generally given by equation (13), where $S_{h}$ is now determined from table 4 . By the multiplication of the matrices in the sequence 
Table 5. Parameters used in the construction of figure 5.

\begin{tabular}{|c|c|c|c|}
\hline$k$ & Coordinate & $\begin{array}{l}\text { String number } \\
\text { (base } B=2 \text { ) }\end{array}$ & $h$ \\
\hline 0 & $(0,0,0)$ & 000 & 0 \\
\hline 1 & $(0,0,1)$ & 001 & 1 \\
\hline 1 & $(0,1,0)$ & 010 & 2 \\
\hline 1 & $(1,0,0)$ & 100 & 4 \\
\hline 2 & $(0,1,1)$ & 011 & 3 \\
\hline 2 & $(1,0,1)$ & 101 & 5 \\
\hline 2 & $(1,1,0)$ & 110 & 6 \\
\hline 3 & $(1,1,1)$ & 111 & 7 \\
\hline
\end{tabular}

$M_{C} M_{E} M_{C} M_{E} M_{C} M_{E} M_{C}$, the order corresponding to the fourth term within the second line of (5), equation (16) becomes

$$
\begin{aligned}
M_{C} M_{E} M_{C} M_{E} & M_{C} M_{E} M_{C}=\hat{\boldsymbol{i}}_{2} S_{1} \hat{\boldsymbol{i}}_{2} S_{2} \hat{\boldsymbol{i}}_{2} S_{3} \hat{\boldsymbol{i}}_{1}+\hat{\boldsymbol{i}}_{2} S_{1} \hat{\boldsymbol{i}}_{2} S_{2} \hat{\boldsymbol{i}}_{1} S_{6} \hat{\boldsymbol{i}}_{2} \\
& +\hat{\boldsymbol{i}}_{2} S_{1} \hat{\boldsymbol{i}}_{1} S_{5} \hat{\boldsymbol{i}}_{2} S_{6} \hat{\boldsymbol{i}}_{2}+\hat{\boldsymbol{i}}_{1} S_{4} \hat{\boldsymbol{i}}_{2} S_{5} \hat{\boldsymbol{i}}_{2} S_{6} \hat{\boldsymbol{i}}_{2} \\
= & \sum_{r, s, t}\left(\frac{\mu_{i}^{0 t} \mu_{j}^{t s} \mu_{k}^{s r} \mu_{l}^{r 0}}{\left(E_{t 0}-3 \hbar c k\right)\left(E_{s 0}-2 \hbar c k\right)\left(E_{r 0}-\hbar c k\right)}\right. \\
& +\frac{\mu_{i}^{0 t} \mu_{j}^{t s} \mu_{l}^{s r} \mu_{k}^{r 0}}{\left(E_{t 0}+\hbar c k\right)\left(E_{s 0}-2 \hbar c k\right)\left(E_{r 0}-\hbar c k\right)} \\
& +\frac{\mu_{i}^{0 t} \mu_{l}^{t s} \mu_{j}^{s r} \mu_{k}^{r 0}}{\left(E_{t 0}+\hbar c k\right)\left(E_{s 0}+2 \hbar c k\right)\left(E_{r 0}-\hbar c k\right)} \\
& \left.+\frac{\mu_{l}^{0 t} \mu_{i}^{t s} \mu_{j}^{s r} \mu_{k}^{r 0}}{\left(E_{t 0}+\hbar c k\right)\left(E_{s 0}+2 \hbar c k\right)\left(E_{r 0}+3 \hbar c k\right)}\right) \\
= & \chi_{i j k l}^{(3)}\left(\omega^{\prime \prime} ; \omega, \omega, \omega\right) .
\end{aligned}
$$

Again, the pre-multiplier $\varepsilon_{0} / \rho$ is required to ensure that this third-order electric susceptibility has the necessary units.

\subsection{Parametric down-conversion}

Another nonlinear process that entails three matter-photon interactions is parametric downconversion (PDC). This involves the conversion of an input photon (with frequency $\omega_{1}$ ) into two emission photons of $\omega_{2}$ and $\omega_{3}$, where $\omega_{1}=\omega_{2}+\omega_{3}$. To enable photon emission at a specified pair of frequencies, since an infinite number of pairs will satisfy conservation of energy, the system is irradiated with a beam of frequency $\omega_{2}$, to allow stimulated emission to occur. Here, the net process is represented by the vector set $I^{\mathrm{PDC}}=\left\{\hat{\boldsymbol{i}}_{1}, \hat{\boldsymbol{i}}_{2}, \hat{\boldsymbol{i}}_{3}\right\}$, in which $\hat{\boldsymbol{i}}_{3}$ signifies a photon annihilation event, and $\hat{\boldsymbol{i}}_{1}$ and $\hat{\boldsymbol{i}}_{2}$ photon emissions with frequencies $\omega_{2}$ and $\omega_{3}$, respectively. The coordinate of each state and the corresponding $k$ and $h$ values are calculated (table 5) and the explicit details of these states are given in table 6 .

In deriving $\chi_{i j k}^{(2)}\left(\omega_{3} ; \omega_{1},-\omega_{2}\right)$, from equation (10), it is found that $j$ now equates to 1 , 2 or 3 -which are related to $\Delta h=4$ ( $k_{3}$ emission), 2 ( $k_{2}$ emission) or 1 (an absorption), respectively-so that

$$
\begin{gathered}
M_{C}=|0\rangle \hat{\boldsymbol{i}}_{3}\langle 1|+| 0\rangle \hat{\boldsymbol{i}}_{2}\langle 2|+| 0\rangle \hat{\boldsymbol{i}}_{1}\langle 4|+| 1\rangle \hat{\boldsymbol{i}}_{2}\langle 3|+| 1\rangle \hat{\boldsymbol{i}}_{1}\langle 5|+| 2\rangle \hat{\boldsymbol{i}}_{3}\langle 3|+| 2\rangle \hat{\boldsymbol{i}}_{1}\langle 6| \\
+|4\rangle \hat{\boldsymbol{i}}_{3}\langle 5|+| 4\rangle \hat{\boldsymbol{i}}_{2}\langle 6|+| 3\rangle \hat{\boldsymbol{i}}_{1}\langle 7|+| 5\rangle \hat{\boldsymbol{i}}_{2}\langle 7|+| 6\rangle \hat{\boldsymbol{i}}_{3}\langle 7| .
\end{gathered}
$$




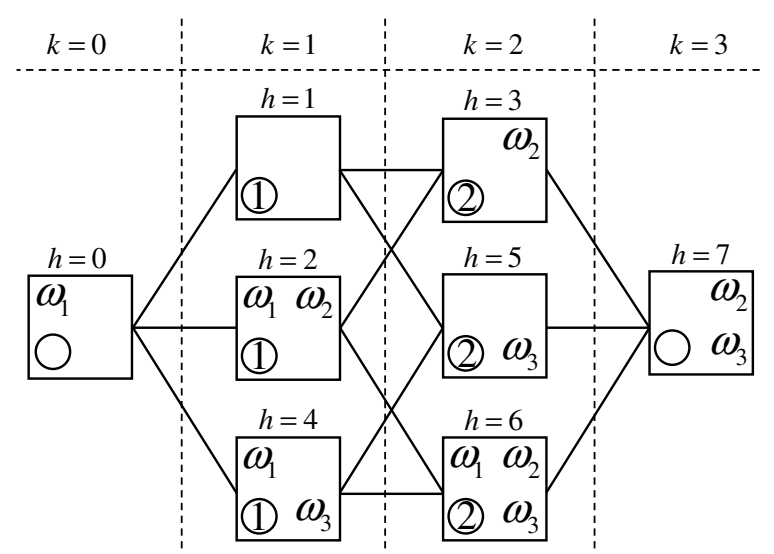

Figure 5. State-sequence diagram for parametric down-conversion. As figure 3, but here with conversion of an input photon (with frequency $\omega_{1}$ ) into two emission photons of $\omega_{2}$ and $\omega_{3}$, where $\omega_{1}=\omega_{2}+\omega_{3}$.

Table 6. All states and their associated energies for the state sequences exhibited in figure 5.

\begin{tabular}{lll}
\hline System state $\left|r_{h}\right\rangle$ & $\left|A_{k} ; n_{\omega_{1}}, n_{\omega_{2}}, n_{\omega_{3}}\right\rangle$ & Energy $E_{r_{h}}$ \\
\hline$|I\rangle=\left|r_{0}\right\rangle$ & $\left|A_{0} ; 1,0,0\right\rangle$ & $E_{0}+\hbar \omega_{1}$ \\
$\left|r_{1}\right\rangle$ & $\left|A_{1} ; 0,0,0\right\rangle$ & $E_{r}$ \\
$\left|r_{2}\right\rangle$ & $\left|A_{1} ; 1,1,0\right\rangle$ & $E_{r}+\hbar\left(\omega_{1}+\omega_{2}\right)$ \\
$\left|r_{4}\right\rangle$ & $\left|A_{1} ; 1,0,1\right\rangle$ & $E_{r}+\hbar\left(\omega_{1}+\omega_{3}\right)$ \\
$\left|r_{3}\right\rangle$ & $\left|A_{2} ; 0,1,0\right\rangle$ & $E_{s}+\hbar \omega_{2}$ \\
$\left|r_{5}\right\rangle$ & $\left|A_{2} ; 0,0,1\right\rangle$ & $E_{s}+\hbar \omega_{3}$ \\
$\left|r_{6}\right\rangle$ & $\left|A_{2} ; 1,1,1\right\rangle$ & $E_{s}+2 \hbar \omega_{1}$ \\
$|F\rangle=\left|r_{7}\right\rangle$ & $\left|A_{0} ; 0 ; 1,1\right\rangle$ & $E_{0}+\hbar \omega_{1}$ \\
\hline
\end{tabular}

Since the route from the initial to final state is a three-step process, the following is determined:

$$
\begin{aligned}
M_{C}^{3} & =\hat{\boldsymbol{i}}_{3} \hat{\boldsymbol{i}}_{2} \hat{\boldsymbol{i}}_{1}+\hat{\boldsymbol{i}}_{2} \hat{\boldsymbol{i}}_{3} \hat{\boldsymbol{i}}_{1}+\hat{\boldsymbol{i}}_{3} \hat{\boldsymbol{i}}_{1} \hat{\boldsymbol{i}}_{2}+\hat{\boldsymbol{i}}_{2} \hat{\boldsymbol{i}}_{1} \hat{\boldsymbol{i}}_{3}+\hat{\boldsymbol{i}}_{1} \hat{\boldsymbol{i}}_{3} \hat{\boldsymbol{i}}_{2}+\hat{\boldsymbol{i}}_{1} \hat{\boldsymbol{i}}_{2} \hat{\boldsymbol{i}}_{3} \\
& =\mu_{k}^{r 0} \mu_{j}^{s r} \mu_{i}^{0 s}+\mu_{j}^{r 0} \mu_{k}^{s r} \mu_{i}^{0 s}+\mu_{k}^{r 0} \mu_{i}^{s r} \mu_{j}^{0 s}+\mu_{j}^{r 0} \mu_{i}^{s r} \mu_{k}^{0 s}+\mu_{i}^{r 0} \mu_{k}^{s r} \mu_{j}^{0 s}+\mu_{i}^{r 0} \mu_{j}^{s r} \mu_{k}^{0 s},
\end{aligned}
$$

where $\hat{\boldsymbol{i}}_{1}, \hat{\boldsymbol{i}}_{2}$ and $\hat{\boldsymbol{i}}_{3}$ correspond to the transition dipole moment components $\mu_{i}, \mu_{j}$ and $\mu_{k}$, respectively. Equation (19) represents a sum of the numerators of the final result, with their corresponding denominators being determined by the application of equation (13) - as earlier, but with $S_{h}$ found from table 6-so that (19) becomes

$$
\begin{aligned}
& M_{C} M_{E} M_{C} M_{E} M_{C}=\hat{\boldsymbol{i}}_{3} S_{1} \hat{\boldsymbol{i}}_{2} S_{3} \hat{\boldsymbol{i}}_{1}+\hat{\boldsymbol{i}}_{2} S_{2} \hat{\boldsymbol{i}}_{3} S_{3} \hat{\boldsymbol{i}}_{1}+\hat{\boldsymbol{i}}_{3} S_{1} \hat{\boldsymbol{i}}_{1} S_{5} \hat{\boldsymbol{i}}_{2}+\hat{\boldsymbol{i}}_{2} S_{2} \hat{\boldsymbol{i}}_{1} S_{6} \hat{\boldsymbol{i}}_{3} \\
&++\hat{\boldsymbol{i}}_{1} S_{4} \hat{i}_{3} S_{5} \hat{\boldsymbol{i}}_{2}+\hat{\boldsymbol{i}}_{1} S_{4} \hat{i}_{2} S_{6} \hat{\boldsymbol{i}}_{3} \\
&= \sum_{r, s}\left(\frac{\mu_{i}^{0 s} \mu_{j}^{s r} \mu_{k}^{r 0}}{\left(E_{s 0}-\hbar \omega_{3}\right)\left(E_{r 0}-\hbar \omega_{1}\right)}+\frac{\mu_{i}^{0 s} \mu_{k}^{s r} \mu_{j}^{r 0}}{\left(E_{s 0}-\hbar \omega_{3}\right)\left(E_{r 0}+\hbar \omega_{2}\right)}\right. \\
&+\frac{\mu_{j}^{0 s} \mu_{i}^{s r} \mu_{k}^{r 0}}{\left(E_{s 0}-\hbar \omega_{2}\right)\left(E_{r 0}-\hbar \omega_{1}\right)}+\frac{\mu_{k}^{0 s} \mu_{i}^{s r} \mu_{j}^{r 0}}{\left(E_{s 0}+\hbar \omega_{1}\right)\left(E_{r 0}+\hbar \omega_{2}\right)}
\end{aligned}
$$




$$
\begin{aligned}
& \left.+\frac{\mu_{j}^{0 s} \mu_{k}^{s r} \mu_{i}^{r 0}}{\left(E_{s 0}-\hbar \omega_{2}\right)\left(E_{r 0}+\hbar \omega_{3}\right)}+\frac{\mu_{k}^{0 s} \mu_{j}^{s r} \mu_{i}^{r 0}}{\left(E_{s 0}+\hbar \omega_{1}\right)\left(E_{r 0}+\hbar \omega_{3}\right)}\right) \\
= & \chi_{i j k}^{(2)}\left(\omega_{3} ; \omega_{1},-\omega_{2}\right) .
\end{aligned}
$$

This equation again excludes the pre-multiplier that ensures the correct unit for the susceptibility.

\section{Conclusion}

The basic principles of nonlinear optics have been rapidly developed and consolidated since the production of the first laser. Most of the original expositions built on the construct of a classical electric polarization, expanded as a power series in the oscillatory input field-a description that is still presented in most textbooks. Typically, nonlinear susceptibilities are derived using this approach, usually assisted by the use of Feynman or analogous diagrams. This standard approach has numerous intricacies, and it proves somewhat arduous even for a relatively simple process. Moreover, it offers little assistance in the development of a fundamental understanding of nonlinear optical response.

In this paper, a more direct and transparent route to the required results has been given, based on quantum electrodynamics. The latter is a theory in which both matter and radiation are treated quantum mechanically; giving legitimacy to the concept of the photon (unlike the traditional formulation), it also elucidates the physics. The graphical representations this method employs, state-sequence diagrams, prove to convey several key advantages over Feynman diagrams: (i) the connectivity of different quantum channels is readily identifiable; (ii) salient parameters for states that feature in several channels need only be computed once; (iii) all time orderings for a particular process are embedded in a single graph; (iv) results associated with higher order corrections are calculable from the same graph without modification.

In summary, we have shown that the presented procedures for characterizing optical nonlinearity in quantum terms are both simple and intuitive. They are readily amenable to advanced physics classes. As well as introducing students to the methods of molecular quantum electrodynamics, these methods afford a means of significantly deepening an appreciation of optical nonlinearity. The given examples of applications to harmonic generation and downconversion illustrate the means of straightforward extension to processes of arbitrary photonic order. The technique we have described represents an obvious scope for class exercises and research project work, comprehensively introducing students to subjects at the heart of modern optics.

\section{Acknowledgments}

Special thanks to Richard G Crisp are due, for his contribution to the novel derivation method presented in this paper. We also thank Luciana Dávila Romero, Jamie Leeder and Justo Rodríguez for helpful comments on the manuscript. Furthermore, we are grateful to the Engineering and Physical Sciences Research Council and Leverhulme Trust for financial support.

\section{References}

[1] Bloembergen N 1996 Nonlinear Optics 4th edn (Singapore: World Scientific)

[2] Butcher P N and Cotter D 1990 The Elements of Nonlinear Optics (Cambridge: Cambridge University Press) 
[3] Jha S S 1995 Perspectives in Optoelectronics (Singapore: World Scientific)

[4] Sauter E G 1996 Nonlinear Optics (New York: Wiley)

[5] He G S and Liu S H 2000 Physics of Nonlinear Optics (Singapore: World Scientific)

[6] Shen Y R 2002 The Principles of Nonlinear Optics (New York: Wiley)

[7] Sutherland R L 2003 Handbook of Nonlinear Optics 2nd edn (New York: Dekker)

[8] Banerjee P P 2003 Nonlinear Optics: Theory, Numerical Modeling, and Applications (New York: Dekker)

[9] Novotny L and Hecht B 2006 Principles of Nano-Optics (Cambridge: Cambridge University Press)

[10] Boyd R W 2008 Nonlinear Optics 3rd edn (New York: Academic)

[11] Loudon R 2000 The Quantum Theory of Light 3rd edn (New York: Oxford University Press)

[12] Chartier G 2005 Introduction to Optics (New York: Springer)

[13] Zernike F and Midwinter J E 2006 Applied Nonlinear Optics (New York: Dover)

[14] Pedrotti F L, Pedrotti L M and Pedrotti L S 2007 Introduction to Optics 3rd edn (Upper Saddle River, NJ: Pearson-Prentice Hall)

[15] Power E A 1964 Introductory Quantum Electrodynamics (London: Longman)

[16] Healy W P 1982 Non-relativistic Quantum Electrodynamics (London: Academic)

[17] Milonni P W 1994 The Quantum Vacuum: An Introduction to Quantum Electrodynamics (New York: Academic)

[18] Andrews D L and Allcock P 2002 Optical Harmonics in Molecular Systems (Weinheim: Wiley)

[19] Ward J F 1965 Rev. Mod. Phys. 371

[20] Andrews D L 1993 Modern Nonlinear Optics, Part 2 (Adv. Chem. Phys. vol 85) ed M W Evans and S Kielich (New York: Wiley) p 545

[21] Andrews D L and Allcock P 2001 Modern Nonlinear Optics, Part 1 (Adv. Chem. Phys. vol 119) ed M W Evans (New York: Wiley) p 603

[22] Andrews D L 1985 A simple statistical treatment of multiphoton absorption Am. J. Phys. 531001

[23] Cohen-Tannoudji C, Dupont-Roc J and Grynberg G 1992 Atom-Photon Interactions: Basic Processes and Applications (New York: Wiley) chapters 1 and 3

[24] Jenkins R D, Andrews D L and Dávila L C 2002 Romero J. Phys. B: At. Mol. Opt. Phys. 35445

[25] Jenkins R D and Andrews D L 1998 J. Phys. Chem. A 10210834

[26] Andrews D L and Jenkins R D 2001 J. Chem. Phys. 1141089

[27] Bradshaw D S and Andrews D L 2005 Phys. Rev. A 72033816

[28] Andrews D L and Crisp R G 2006 J. Opt. A: Pure Appl. Opt. 8 S106

[29] Alligood B W and Salam A 2007 Mol. Phys. 105395

[30] Weisstein E W 2002 CRC Concise Encyclopaedia of Mathematics 2nd edn (Boca Raton, FL: Chapman and Hall/CRC) 